\title{
Revue Entwicklungsethnologie : Zeitschrift der Arbeits Gemeinschaft Entwicklungsenthnologie e.V. (AGEE)
}

Katrin Langewiesche

\section{CpenEdition}

Journals

Édition électronique

URL : http://journals.openedition.org/apad/1681

DOI : 10.4000/apad. 1681

ISSN : 1950-6929

Éditeur

LIT Verlag

Édition imprimée

Date de publication : 1 juin 1995

Référence électronique

Katrin Langewiesche, «Revue Entwicklungsethnologie : Zeitschrift der Arbeits Gemeinschaft Entwicklungsenthnologie e.V. (AGEE) », Bulletin de l'APAD [En ligne], 9 | 1995, mis en ligne le 26 juillet 2007, consulté le 24 septembre 2020. URL : http://journals.openedition.org/apad/1681 ; DOI : https:// doi.org/10.4000/apad.1681

Ce document a été généré automatiquement le 24 septembre 2020.

Bulletin de I'APAD 


\title{
Revue Entwicklungsethnologie : Zeitschrift der Arbeits Gemeinschaft Entwicklungsenthnologie e.V. (AGEE)
}

\author{
Katrin Langewiesche
}

1 La revue Entwicklungsethnologie est centrée autour des thèmes de l'anthropologie du développement Elle paraît depuis 1992 deux fois par an.

2 L'initiative pour la publication d'une telle revue est née dans un groupe de travail de l'association d'ethnologie allemande (Deutsche Gesellschaft für Volkerkunde) qui s'est intéressé à l'anthropologie du développement Constatant le manque de communication entre les chercheurs et les praticiens dans le milieu du développement, les membres de ce groupe de travail (Arbeitsgemeinschaft Entwicklungsethnologie) ont crée la revue Entwicklungsenthnologie dont le but est d'être un lieu de discussion pour ces deux groupes. Les membres du groupe de travail et fondateurs de la revue sont essentiellement des anthropologues et des chercheurs d'autres sciences sociales, mais les contributions sont, suivant l'objectif de la revue, bien reparties entre praticiens, chercheurs et anthropologues-experts.

3 La tendance commune aux différents articles est de définir la compétence anthropologique dans le domaine du développement D'une manière générale on peut différencier trois genres d'articles :

4 1.Contributions méthodologiques

5 Les auteurs présentent des méthodes d'enquête rapides (i.e. Bierschenk et Olivier de Sardan, 1995/1; Vokral, 1994/1) ou des stratégies nouvelles (i.e. Schmid-Heregeth, 1995/1 ; Pottier, 1993/2).

6 2. Descriptions et analyses des projets de développement

7 Les auteurs présentent des conceptions de projets particuliers (i.e. Huba-Mang, 1995/1 ; Dietz, 1995/1 ; Rodenberg 1994/2) ou des expériences personnelles (Le. Meyer, 1994/2). 
Les publications couvrent des aires géographiques comme l'Amérique Latine, l'Afrique et l'Asie.

8 3. Réflexions théoriques autour des différents sujets de l'anthropologie du développement

9 Pour ne citer que quelques exemples : Schafer (1994/2) écrit sur "les femmes et le développement", Drubig (1994/1) réfléchit sur le rôle du chercheur occidental dans le "Tiers Monde", Sommerfeld (1995/1) sur la nécessité d'un travail interdisciplinaire entre différentes disciplines dans le milieu du développement, etc...

10 Les articles sont publiés en anglais ou en allemand avec un résumé en deux langues au début de chaque article. A la fin de chaque contribution, on trouve, ce qui est très agréable pour des lecteurs qui ne sont pas du milieu du développement, une brève présentation de l'auteur.

11 Chaque numéro contient cinq à sept articles, puis une rubrique" discussion", des comptes-rendus d'ouvrages et des annonces divers. Tous, bien sûr, centrés autour de l'anthropologie du développement.

12 Les éditeurs de la revue Entwicklungsethnologie adoptent explicitement une optique d'anthropologie appliquée. La revue atteint les objectifs de ses éditeurs en donnant effectivement un bon aperçu de la rencontre entre chercheurs et praticiens dans le milieu du développement. Elle montre les possibilités d'une telle rencontre mais aussi ses limites. Nombre d'articles restent superficiels au niveau méthodologique ou théorique. En outre, la présentation des nouvelles stratégies mériterait souvent un approfondissement.

13 Mais les éditeurs ont réussi à créer un lieu de discussion à mi-chemin entre le monde de la science et celui de la pratique, bien que le scepticisme concernant l'articulation possible de la théorie et de la pratique dans le domaine du développement se maintienne. 